Research Article

Mayeul Arminjon*

\title{
On the equations of electrodynamics in a flat or curved spacetime and a possible interaction energy
}

https://doi.org/10.1515/phys-2018-0065

Received May 23, 2018; accepted Jun 18, 2018

\begin{abstract}
In this paper the independent equations of continuum electrodynamics and their quantity are investigated, beginning with the standard equations used in special and general relativity. Using differential identities it is checked that there are as many independent equations as there are unknowns, for the case with given sources as well as for the general case where the motion of the charged medium producing the field is unknown. This problem is then discussed in an alternative theory of gravity with a preferred reference frame, in order to constrain an additional, "interaction" energy tensor that has to be postulated in this theory, and that would be present also outside usual matter. In order that the interaction tensor be Lorentz-invariant in special relativity, it has to depend only on a scalar field $p$. Since the system of electrodynamics is closed in the absence of the interaction tensor, just one scalar equation more is needed to close it again in the presence of $p$. That equation is taken to be the equation for charge conservation. Finally, the equations that allow the determination of field $p$ are derived in a given weak gravitational field and in a given electromagnetic field.
\end{abstract}

Keywords: Maxwell equations, special relativity, general relativity, alternative theory of gravitation, preferred reference frame

PACS: 04.25.Nx, 04.40.-b, 04.50.-h, 98.58.Ay

\section{Introduction and summary}

The usual approach to classical electrodynamics considers both a system of point charges and the electromagnetic (EM) field that is produced by them and acts upon

\footnotetext{
^Corresponding Author: Mayeul Arminjon: Univ. Grenoble Alpes, CNRS, Grenoble INP, 3SR, F-38000 Grenoble, France; Email: mayeul.arminjon@3sr-grenoble.fr
}

them [1, 2]. The ideal notion of point charge leads to difficulties such as self-force, infinite energy and run-away solutions (among others) but in the Maxwell equations the charge and current distribution is preferably a continuous one. From the definitions of the "free" densities of electric charge and current [2], it certainly follows that the distribution should be continuous for the macroscopic Maxwell equations (which contain the displacement field $\mathbf{D}$ and the magnetizing field $\mathbf{H}$ in addition to the macroscopic electric field $\mathbf{E}$ and the macroscopic magnetic field $\mathbf{B}$ ). For a linear and isotropic response, the macroscopic equations have the same form as the "microscopic" Maxwell equations (i.e. those defined using the microscopic electric field $\mathbf{E}$ and the microscopic magnetic field B) [2]. Hence, the Maxwell equations without $\mathbf{D}$ and without $\mathbf{H}$, for a continuous charged medium and its EM field, will be considered. This applies to a macroscopic, practical situation, for a linear and isotropic medium. This applies also to the general situation at a "microscopic but still classical" scale, when considering the total microscopic charge density and the total microscopic current density, without any distinction between "free" and "bound" charges. However, in the case that the source is not considered known, it is assumed that the velocity of the electric current is the velocity of the charged medium, see Eqs. (19) and (23) below.

The focus of this investigation is the development of continuum electrodynamics in an alternative, scalar theory of gravitation with a preferred reference frame or "ether", in short the scalar ether theory or SET. An alternative extension of Maxwell's second group to the situation with gravitation, consistent with SET, had been proposed in a previous work [3]. That alternative second group predicted charge non-conservation in a variable gravitational field. However, in a later work [4], it has been found that the charge production/destruction predicted appears too high. It has also been found [4] that the cause for this failure is that in SET one should not assume that the total energy(-momentum-stress) tensor $\boldsymbol{T}$ (whose $T^{00} \mathrm{com}$ ponent in the preferred frame is the source of the gravitational field [5]) is the sum of the energy tensors of the 
charged medium and the EM field: $\boldsymbol{T} \neq \boldsymbol{T}_{\text {chg }}+\boldsymbol{T}_{\text {field }}$. The primary aim of this work was therefore to derive explicit equations that would allow the "interaction energy tensor" to be calculated

$$
\boldsymbol{T}_{\text {inter }}:=\boldsymbol{T}-\boldsymbol{T}_{\text {chg }}-\boldsymbol{T}_{\text {field }}
$$

in relevant situations. This is of interest not only for SET, but also because it might be the case that the interaction energy contribute to the "dark matter" [4]. To assess $\boldsymbol{T}_{\text {inter }}$ the constraints which are imposed on it must be defined, hence a detailed investigation into which are the independent equations of continuum electrodynamics and their quantity is required.

It is necessary to begin with the case of special relativity (SR) and general relativity (GR). This will be addressed in Section 2. There it is shown that the notion of differential identity allows a simple explanation of why the eight components of the standard Maxwell equations are needed to determine the six components of $\mathbf{E}$ and $\mathbf{B}$, when the 4current is given; and this same notion shows that the more complete system obtained by adding the equation of motion of the charged continuum is closed, too. (For that system the 4-current is an unknown.)

The rest of the paper addresses the electrodynamics of SET and the equations for the interaction tensor. Section 3 summarizes the situation [3] when the "additivity assumption" (equivalent to $\boldsymbol{T}_{\text {inter }}=\mathbf{0}$ in (1)) is made, and, using the same method as in Sect. 2, it is shown that the equations form a closed system of PDE's. Section 4 briefly exposes the reasons [4] that counter this assumption. Then Sect. 5 studies the constraints that are imposed on $\boldsymbol{T}_{\text {inter }}$. The demand that it be Lorentz-invariant in SR leads to the simple form (43), depending on a scalar field $p$. One scalar equation is then lacking in the electrodynamics of SET, due to the introduction of one new unknown $p$. It follows that Maxwell's second group cannot be the same in "SET with $p^{\prime \prime}$ as it is in GR and in the other metric theories of gravitation. In Sect. 6, the system of electrodynamics in "SET with $p$ " is closed by adding the charge conservation. The field $p$ is shown to be constant (and arguably zero) for a gravitational field that is constant in the preferred reference frame assumed by that theory. Hence, $p$ is constant, and arguably zero, in SR with this system of equations. Finally, Sect. 7 establishes the explicit equation that determines the field $p$ in a weak and slowly varying gravitational field, Eq. (75), and proposes an integration procedure to derive the numerical values of $p$.

\section{Independent equations in standard theory}

"Standard theory", is defined here as the electrodynamics in GR, or possibly in another metric theory of gravity. This includes SR as the case that the spacetime metric is Minkowski's. In any such theory, the dynamical equation verified by the total energy(-momentum-stress) tensor $\boldsymbol{T}$ of matter and non-gravitational fields is

$$
T_{; v}^{\mu \nu}=0
$$

and, more generally, there is a rule to go from any equation valid in SR in Cartesian coordinates to an equation valid with a general Lorentzian metric in general coordinates: "comma goes to semicolon" [6], i.e., partial derivatives are replaced by covariant derivatives based on the connection associated with the spacetime metric $\gamma$. (This rule becomes ambiguous in cases with derivatives of order larger than one, but this can be avoided in the case of the Maxwell equations: they involve only the EM field tensor $\boldsymbol{F}$, not the EM 4-potential A.) This leads to the standard equations for the Maxwell field in a curved spacetime: the first group,

$$
F_{\lambda \mu, v}+F_{\mu v, \lambda}+F_{v \lambda, \mu}=F_{\lambda \mu ; \nu}+F_{\mu \nu ; \lambda}+F_{v \lambda ; \mu}=0
$$

(the first equality is an identity due to the antisymmetry of $\boldsymbol{F}\left(F_{\mu \nu}=-F_{\nu \mu}\right)$ and to the symmetry of the metric connection), and the second group, ${ }^{1}$

$$
F_{; v}^{\mu v}=-\mu_{0} J^{\mu}
$$

(Here $J^{\mu}(\mu=0, \ldots, 3)$ are the components of the EM 4current $\mathbf{J}$ and $\mu_{0}$ is the permeability of free space.) Now the number of independent unknowns and independent equations must be assessed. First, since the first group can be written as

$$
M_{\lambda \mu \nu}:=F_{\lambda \mu ; \nu}+F_{\mu \nu ; \lambda}+F_{\nu \lambda ; \mu}=0,
$$

and since the 1.h.s. is antisymmetric, i.e., $M_{\lambda \mu \nu}=-M_{\mu \lambda \nu}=$ $-M_{\lambda v \mu}$, it is usual to note that (3) contains exactly four linearly-independent equations: for example, $M_{012}=0$, $M_{013}=0, M_{023}=0, M_{123}=0$. Also, the four equations in the second group (4) are linearly independent.

1 In this paper, as in the foregoing [4], we are using the SI units and the (+ - - ) signature. (In Refs. $[1,3,13]$, the Gauss units were used.) Greek indices go from 0 to 3, Latin ones from 1 to 3. Indices are raised or lowered using the spacetime metric $\gamma$. 


\subsection{Case with given 4-current}

For the solutions to the Maxwell equations, the 4-current $\mathbf{J}$ is often considered given. This results in eight Maxwell equations (3)-(4) for the six independent unknowns $F_{\mu \nu}(0 \leq \mu<v \leq 3)$. It is known that those eight equations are needed, i.e., one cannot consistently remove two of them from the whole. In particular, one cannot consistently remove the two divergence equations $\operatorname{div} \mathbf{B}=0$ and $\operatorname{div} \mathbf{E}=\rho_{\mathrm{el}} / \epsilon_{0}$ from the usual three-vector form of the flatspacetime Maxwell equations [7-9]. ${ }^{2}$ In this regard, it is observed [7] that the Maxwell equations form two pairs of "div-curl" systems of equations, a div-curl system consisting of four scalar equations for three scalar unknowns $u^{i}(i=1,2,3)$ :

$$
\operatorname{div} \mathbf{u}=f, \quad \operatorname{rot} \mathbf{u}=\mathbf{s} .
$$

From this, the following has been shown in a detailed mathematical work [7]: (i) There is a theorem stating uniqueness of the solutions $\mathbf{u}$ to the div-curl system, modulo suitable boundary conditions, and provided the data $\mathbf{s}$ verifies the compatibility condition

$$
\operatorname{div} \mathbf{s}=0
$$

(which follows from applying the differential identity $\operatorname{div} \operatorname{rot} \mathbf{u} \equiv 0$ to Eq. $(6)_{2}$ ). (ii) Removing the divergence equation in one div-curl system leads to a system whose solutions are not necessarily solutions of that div-curl system.

However, it is proposed that the fact that the div-curl system, or respectively the Maxwell equations, are not overdetermined, is more easily understood from the observation that these systems of PDE's verify definite differential identities. This notion (without its name) is recognized by Liu [9] as relevant (see his Definition II), but no indication is provided of which differential identities do apply to the div-curl system (6) or respectively to the Maxwell equations. The identity div rot $\mathbf{u} \equiv 0$, which he mentions, is not a differential identity of the div-curl system (6), because $\operatorname{rot} \mathbf{u}=\mathbf{0}$ does not belong to the equations of that system (unless $\mathbf{s} \equiv \mathbf{0}$ ). Also, Eq. (7) is not a differential identity, i.e., a partial differential equation that is satisfied by any regular vector function s. If Eq. (7) is satisfied by some par-

2 Here $\rho_{\mathrm{el}}$ is the volume density of the electric charge and $\epsilon_{0}$ is the permittivity of the vacuum. With the SI units and the (+ - - ) signature, the relation between the field tensor $\boldsymbol{F}$ and the electric and magnetic fields $\mathbf{E}$ and $\mathbf{B}$ in Cartesian coordinates in a Minkowski spacetime is given on the Wikipedia page "Electromagnetic tensor", or by Eq. (47) of Ref. [4]. ticular function $\mathbf{s}$, then the following is a differential identity, i.e., it is valid for any regular vector function $\mathbf{u}$ :

$$
\operatorname{div}(\operatorname{rot} \mathbf{u}-\mathbf{s}) \equiv 0 .
$$

This is a differential identity of the system (6), i.e., it has the form

$$
\sum_{k=1}^{n} \mathcal{O}_{k} \mathcal{P}_{k} \mathbf{u} \equiv 0
$$

where $\mathcal{P}_{k} \mathbf{u}=0(k=1, \ldots, n$, thus $n=4$ for the system (6)) are the scalar PDE's of the system, and where $\mathcal{O}_{k}$ are scalar differential operators of the first order, or the zero operator. Some among the $\mathcal{O}_{k}$ 's, but not all, can be the zero operator, meaning that the corresponding equation $\mathcal{P}_{k} \mathbf{u}=0$ is not involved in the differential identity. (Also, $\mathbf{u}$ designates in general the list of unknown functions in the system.) By definition, a differential identity like (9) has to apply whether or not any of the equations of the system $\left(\mathcal{P}_{k} \mathbf{u}=0\right)$ is satisfied. In view of the scalar identity (8), the system (6) has only three independent equations instead of four. But since it is a differential identity, not an algebraic one, and since that identity involves several equations among the scalar PDE's of the system, therefore none of the scalar PDE's of the system can be removed without altering it. Note that (under the validity of the compatibility condition (7)), the differential identity (8) is already a differential identity of the "curl system" $(6)_{2}$ alone, which is an underdetermined system: $3-1=2$ independent equations for 3 unknowns. It thus becomes obvious that, if one removes the divergence equation $(6)_{1}$, one will indeed be able to find "spurious" solutions, i.e., undesired ones.

Consider now the flat-spacetime Maxwell system in vacuo: Eqs. (4)-(5) with commas instead of semicolons (in Cartesian coordinates for the Minkowski metric), and with $J^{\mu}=0$. As noted by Das [10], this system has two differential identities

$$
S:=\varepsilon_{\mu \nu \rho \sigma} M_{\mu \nu \rho, \sigma} \equiv 0
$$

(where $\varepsilon_{\mu \nu \rho \sigma}$ is the signature of the permutation $(\mu \nu \rho \sigma)$ of $\{0, \ldots, 3\})$, and

$$
F_{, v, \mu}^{\mu v} \equiv 0,
$$

both result (after a short algebra, for (10)) from the antisymmetry of the tensor $\boldsymbol{F}$. The first identity is a differential identity of the first Maxwell group (5), the second one is a differential identity of the second Maxwell group in vacuo. Thus there are six independent equations for the six unknowns.

Finally, consider the Maxwell system in a general spacetime (4)-(5). In a general coordinate system in a general Lorentzian spacetime, similarly with (10) and (11) the 
two independent differential identities are

$$
e^{\mu \nu \rho \sigma} M_{\mu \nu \rho ; \sigma} \equiv 0
$$

(where $e_{\mu \nu \rho \sigma}$ is the antisymmetric tensor that coincides with $\varepsilon_{\mu \nu \rho \sigma}$ in coordinates such that the natural basis is direct and that $\left.\gamma:=\operatorname{det}\left(\gamma_{\mu \nu}\right)=-1\right)$, and

$$
F_{; v ; \mu}^{\mu v} \equiv 0 .
$$

These identities apply (whether or not Eqs. (4) or (5) are verified), since the 1.h.s. of either (12) or (13) is a manifestly invariant scalar, and is zero (as for (10) or (11)) in coordinates such that, at the event considered, the Christoffel symbols vanish and the matrix $\left(\gamma_{\mu \nu}\right)=\left(\eta_{\mu v}\right):=$ $\operatorname{diag}(1,-1,-1,-1)$. The first, Eq. (12), is a differential identity of the first Maxwell group (5). The identity (13) implies that the second Maxwell group (4) has charge conservation as a compatibility condition:

$$
J_{; \mu}^{\mu}=0 .
$$

Hence, it is here, as with the div-curl system (6): if the compatibility condition (14) is satisfied, then from (13) the following differential identity of the second Maxwell group (4) is derived:

$$
S^{\prime}:=\left(F_{; v}^{\mu v}+\mu_{0} J^{\mu}\right)_{; \mu} \equiv 0 .
$$

There are therefore six independent equations for the six unknowns.

It is useful to show how the identities (12) and (15) appear with the 3-vector form of the Maxwell equations for a flat spacetime. Using Eqs. (24.13) and (24.14) of Fock [13], the l.h.s. of the identity (10) can be rewritten as

$$
S=6\left[\operatorname{div}\left(\operatorname{rot} \mathbf{E}+\frac{\partial \mathbf{B}}{\partial t}\right)-\frac{\partial}{\partial t}(\operatorname{div} \mathbf{B})\right] .
$$

In a flat spacetime, the identity $S \equiv 0$ is hence a differential identity of the 3-vector form (e.g. Eqs. (1) and (4) in Ref. [9]) of Maxwell's first group. In a flat spacetime, the identity (15) rewrites, using $\mu_{0} \epsilon_{0}=1 / c^{2}$, as the following differential identity of the usual form of Maxwell's second group:

$$
\begin{aligned}
-S^{\prime} & =\operatorname{div}\left[\operatorname{rot} \mathbf{B}-\mu_{0}\left(\mathbf{j}+\epsilon_{0} \frac{\partial \mathbf{E}}{\partial t}\right)\right] \\
& +\mu_{0} \epsilon_{0} \frac{\partial}{\partial t}\left(\operatorname{div} \mathbf{E}-\frac{\rho_{\mathrm{el}}}{\epsilon_{0}}\right) \equiv 0 .
\end{aligned}
$$

This identity is satisfied provided that the integrability condition of Maxwell's second group: Eq. (14) or equivalently $\frac{\partial \rho_{\mathrm{el}}}{\partial t}+\operatorname{div} \mathbf{j}=0$, is satisfied (here $\mathbf{j}$ is the 3 -vector with components $j^{i}:=J^{i}$ ). As for $S \equiv 0$, the identity (17) applies whether or not any of the Maxwell equations is satisfied.

\subsection{Complete system for a deformable charged medium}

The 4-current density $\mathbf{J}$ can be considered given (as an approximation), as above. This is not the general case, however. A continuous charged medium is subjected to the Lorentz 4-force (density)

$$
f^{\mu}:=F^{\mu}{ }^{\nu} J^{v},
$$

which modifies the (3-)velocity field $\mathbf{v}$ of the charged medium (especially if the latter is deformable, as is the case e.g. in magnetohydrodynamics), and hence the current $\mathbf{J}$ also. In any coordinates $x^{\mu}$, the components of the latter 4-vector are defined as ([1], Eq. (90.3)):

$$
J^{\mu}:=\frac{\rho_{\mathrm{el}}}{\beta} \frac{\mathrm{d} x^{\mu}}{\mathrm{d} t},
$$

where

$$
\beta:=\sqrt{\gamma_{00}},
$$

and $t:=x^{0} / c$. The velocity field $\mathbf{v}$ (whose value at an event $X$ is the velocity of that infinitesimal volume element of the continuous medium which is at $X$ ) is measured with the local standards. This field thus has components $v^{i}=\mathrm{d} x^{i} / \mathrm{d} t_{\mathbf{x}}$ where $t_{\mathbf{x}}$ is the synchronized local time in the reference fluid $\mathcal{F}$ that is considered $[1,11,12]$. (Note that the data of one coordinate system $\left(x^{\mu}\right)$ automatically defines a unique reference fluid, whose reference world lines are the lines $\mathbf{x}:=\left(x^{i}\right)=$ Constant [12].) Assuming that $\mathcal{F}$ has adapted coordinates that verify the synchronization condition

$$
\gamma_{0 i}=0
$$

(and using any such coordinates), we have

$$
\frac{\mathrm{d} t_{\mathbf{x}}}{\mathrm{d} t}=\beta(t, \mathbf{x}),
$$

so that $J^{i}=\rho_{\mathrm{el}} v^{i}$, or

$$
\mathbf{j}=\rho_{\mathrm{el}} \mathbf{V}
$$

for the 3-current, a spatial vector field in the reference fluid $\mathcal{F}$. A deformable charged medium has state parameters: at least the proper rest-mass density field $\rho^{\star}$ or (equivalently for a barotropic perfect fluid) the pressure field $P$.

In the general case, in addition to the six field unknowns $F_{\mu \nu}(0 \leq \mu<v \leq 3)$, there are also the field unknowns $\rho_{\mathrm{el}}$ and $\mathbf{v}$, plus the other state parameters, say merely the field $\rho^{*}$ for the simplicity of discussion thus five additional unknowns more, that is $6+5=11$ unknowns. The additional equation with respect to the Maxwell equations (4)-(5) is the dynamical equation of GR for the charged medium subjected to the 4-force (18):

$$
T_{\text {chg; } ; \nu}^{\mu \nu}=F_{\lambda}^{\mu} J^{\lambda},
$$


where $\boldsymbol{T}_{\text {chg }}$ is the energy tensor of the deformable charged medium, depending on $\mathbf{v}$ and $\rho^{\star}$. Equation (24) extends a standard equation valid in SR (e.g. [1], Eq. (33.9)), by using the "comma goes to semicolon" rule. It can also be derived from the dynamical equation for the total energy tensor (2), Maxwell's second group (4), and the assumption of additivity of the energy tensors:

$$
\boldsymbol{T}=\boldsymbol{T}_{\text {chg }}+\boldsymbol{T}_{\text {field }},
$$

with $\boldsymbol{T}_{\text {field }}$ the energy tensor of the EM field [1, 13]:

$$
T_{\text {field }}^{\mu v}:=\left(-F_{\lambda}^{\mu} F^{v \lambda}+\frac{1}{4} \gamma^{\mu \nu} F_{\lambda \rho} F^{\lambda \rho}\right) / \mu_{0} .
$$

Indeed, by using the identity $[3,4]$

$$
\mu_{0} T_{\text {field } ; v}^{\mu \nu} \equiv-F_{\lambda}^{\mu} F_{; v}^{v \lambda},
$$

one deduces from the second group (4):

$$
T_{\text {field ; } \nu}^{\mu \nu}=-F_{\lambda}^{\mu} J^{\lambda} \text {. }
$$

Equations (2) and (25) then imply immediately (24). Thus, five unknowns more than in the case with given 4-current, and merely four equations (24) more, in addition to (4) and (5). ${ }^{3}$ However, since in the system [(4)-(5), (24)] the current $\boldsymbol{J}$ is unknown as well as is the EM field $\boldsymbol{F}$, Eq. (15) is not a differential identity of that system, even though it does have the form (9) (with $\mathbf{u}$ denoting the set of all unknowns of the system: now $\mathbf{J}, \boldsymbol{F}$, and $\rho^{*}$, or equivalently $\rho_{\mathrm{el}}, \mathbf{v}, \boldsymbol{F}$, and $\left.\rho^{\star}\right)$. This is because (15), as well as the charge conservation (14), applies only on the solution space of the system. (Both apply there, due to Maxwell's second group (4) and to the identity (13).) Whereas, a differential identity of the system has to be valid for any regular field unknowns $\mathbf{J}, \boldsymbol{F}$, and $\rho^{*}$. Note also that the identity (13) is not a differential identity of the system either, because it does not have the form (9). Thus, for the system [(4)-(5), (24)], only the identity (12) counts as a dependence relation. Providing $12-1=11$ equations for the 11 unknowns.

Note that the above applies in SR as well as in a metric theory of gravitation. However, in the case with gravitation, strictly speaking, the metric field $\gamma$ should not be considered given, since the EM energy tensor does contribute to the total energy tensor and hence influences the metric non-linearly through the Einstein equations (considering GR for definiteness) - even if this influence is very small

3 At least if one assumes a perfect fluid, the mass conservation can be exactly deduced from (24), in nearly the same way as one deduces it from (2) [14] in the case without the EM field and current (in both cases, mass conservation applies iff the fluid is isentropic). Hence it is not an additional equation. in most conditions (e.g. in the solar system). Ten additional unknowns $\gamma_{\mu \nu}(0 \leq \mu \leq v \leq 3)$ must be added to account for this. And ten additional Einstein equations (of which only six count as independent due to the four differential identities of Bianchi), plus the four equations of the gauge condition which is selected.

\section{Independent equations in SET without interaction tensor}

In SET, motion is governed by an extension of the specialrelativistic form of Newton's second law to a curved spacetime, written in the preferred reference fluid $\mathcal{E}$ assumed by the theory. This extension, which is formulated primarily for a test particle [15], can be applied to each particle of a dust, that is an ideal continuum made of a myriad of coherently moving test particles. This leads [3] to the following dynamical equation in the presence of a field of external (3)force with volume density f, components $f^{i}(i=1,2,3)$ :

$$
T_{\text {medium ; }}^{\mu \nu}=b^{\mu}\left(\boldsymbol{T}_{\text {medium }}\right)+f^{\mu}, \quad f^{0}:=\frac{\mathbf{f . v}}{c \beta},
$$

where $\beta$ is defined in Eq. (20), $\mathbf{v}$ is the velocity field (with the local time, see after that equation), $\boldsymbol{T}_{\text {medium }}$ is the energy-momentum tensor of the continuous medium, and

$$
b^{0}(\boldsymbol{T}):=\frac{1}{2} \gamma^{00} g_{i j, 0} T^{i j}, \quad b^{i}(\boldsymbol{T}):=\frac{1}{2} g^{i j} g_{j k, 0} T^{0 k} .
$$

Here $g_{i j}$ are the components of the spatial metric tensor $\mathbf{g}=\mathbf{g}_{\varepsilon}$ associated with the spacetime metric $\gamma$ in the reference fluid $\varepsilon[1,3,17]$. Equation (29) is then assumed to be valid for any continuous medium [3], provided a velocity field can be unambiguously defined for that medium. In the case that the continuous medium is a charged medium and $\mathbf{f}$ is the Lorentz (3-)force, the logic of the theory leads to a definition of its components $f^{i}$ as the spatial components $\mu=i$ in Eq. (18) above [3]. The $f^{0}$ component is thus derived (at least for a dust) to $(29)_{2}$ here, and is also equal to the $\mu=0$ component in Eq. (18) [3] - although the dynamics is different from that of GR. So for a charged medium the dynamical equation is:

$$
T_{\text {chg; } ; v}^{\mu v}=b^{\mu}\left(\boldsymbol{T}_{\text {chg }}\right)+F^{\mu}{ }_{v} J^{v}
$$

The dynamical equation for the total energy tensor $\boldsymbol{T}$ is also obtained by induction from the dust, this time without any non-gravitational external force, and is thus [16]:

$$
T_{; v}^{\mu v}=b^{\mu}(\boldsymbol{T}) .
$$


Often additivity of the energy tensors is assumed, Eq. (25). However, together with the dynamical equations for the charged medium and the total energy tensor, Eqs. (31) and (32), this determines Maxwell's second group. This is already true for GR, as seen by reverting the line of reasoning in Eqs. (27) and (28) above: starting from the dynamical equations for the charged medium and the total energy tensor in GR, Eqs. (24) and (2), and using the additivity (25), we obtain immediately Eq. (28). Equating with the identity (27) gives

$$
-F_{\lambda}^{\mu} F_{; v}^{v \lambda}=-\mu_{0} F_{\lambda}^{\mu} J^{\lambda}
$$

which, for the (generic) case of an invertible matrix $\left(F_{\lambda}^{\mu}\right)$, is equivalent to the second group of GR, Eq. (4). For SET, this same argument, with Eqs. (31) and (32) replacing Eqs. (24) and (2) of GR, leads first to

$$
T_{\text {field ; }}^{\mu \nu}=b^{\mu}\left(\mathbf{T}_{\text {field }}\right)-F^{\mu}{ }_{v} J^{\nu},
$$

from which one gets by (27) [3]:

$$
F_{\lambda}^{\mu} F_{; \nu}^{\lambda \nu}=\mu_{0}\left[b^{\mu}\left(\mathbf{T}_{\text {field }}\right)-F_{\lambda}^{\mu} J^{\lambda}\right] \text {, }
$$

which is the second group from SET when additivity is assumed (25).

The above implies that, for SET with the additivity assumption (25), the system of equations governing the motion of a charged medium and its EM field is: Maxwell's first group (5), plus the dynamical equations for the charged medium and the total energy tensor, Eqs. (31) and (32) or, equivalently, Maxwell's first group (5), plus Eqs. (31) and (35). The unknown fields are the same as for GR: $F_{\mu \nu}(0 \leq \mu<\nu \leq 3), \rho_{\mathrm{el}}$ and $\mathbf{v}$, and $\rho^{*}$, or equivalently $F_{\mu \nu}(0 \leq \mu<\nu \leq 3)$, J, and $\rho^{\star}-11$ unknowns. And as in GR, only the identity (12) is a dependence relation. So again $12-1=11$ equations for the 11 unknowns. To account for the fact that the gravitational field is not given, a scalar gravitational field unknown is added, $\psi:=-\log \beta$, along with the scalar flat-spacetime wave equation obeyed by $\psi$ according to that theory [5].

\section{Interaction energy tensor in SET}

There are three reasons [4] which counter the solution of closing the electrodynamics for SET using the additivity assumption (25):

(i) The version (35) of Maxwell's second group leads to the non-conservation of charge in a variable gravitational field. (This is seen by using the identity (13) [3].) The amounts of electric charge which are predicted to be produced or destroyed in a realistic EM and gravitational field, appear too high to be a tenable prediction. (ii) The energy tensors of the charged medium and the EM field are both non-zero. This results in the presence of a mixture. According to the standard theory of mixtures (which has been developed for non-relativistic physics), the effective energy tensor of the mixture is not the sum of the energy tensors of its constituents [18]. This is not compatible with the additivity assumption (25).

(iii) Equation (34) means that the EM field continuum verifies the dynamical equation (29) for a continuous medium with an external force field $\mathbf{f}_{\text {field }}$ acting on it, with, specifically:

$$
f_{\text {field }}^{\mu}=-f^{\mu}:=-f_{\text {chg }}^{\mu}:=-F^{\mu}{ }_{v} J^{v} .
$$

Using the spatial part of this $(\mu=1,2,3)$, and noting respectively $\mathbf{v}_{\text {field }}$ and $\mathbf{v}_{\text {chg }}$ the velocity fields of the field continuum and the charged continuum (assuming $\mathbf{v}_{\text {field }}$ is well defined), the "time" part rewrites as [4]:

$$
\mathbf{f}_{\text {chg }} \cdot\left(\mathbf{v}_{\text {field }}-\mathbf{v}_{\text {chg }}\right)=0 .
$$

However, for a general EM field, it is not easy to tell how to define its velocity field $\mathbf{v}_{\text {field }}$, be it experimentally or in terms of the energy tensor $\boldsymbol{T}_{\text {field }}$. Therefore, Eq. (37) looks problematic for a general EM field. For a "null" field (i.e., such that the classical invariants are both zero), the velocity field can be defined naturally in terms of the energy tensor $\boldsymbol{T}_{\text {field }}$ [4], and it has modulus $v_{\text {field }}:=$ $\left(\mathbf{g}\left(\mathbf{v}_{\text {field }}, \mathbf{v}\right)_{\text {field }}\right)^{1 / 2}=c$. Furthermore, one can then prove [4] that indeed (37) is satisfied (although usually $v_{\text {chg }} \ll c$ ), but this proof depends heavily on the fact the EM field is a null field.

Therefore the additivity assumption (25) is disregarded. This means that the total tensor $\boldsymbol{T}$ verifying Eq. (32) involves another part, say $\boldsymbol{T}_{\text {inter }}$ :

$$
\boldsymbol{T}=\boldsymbol{T}_{\text {chg }}+\boldsymbol{T}_{\text {field }}+\boldsymbol{T}_{\text {inter }} .
$$

While introducing that "interaction tensor" $\boldsymbol{T}_{\text {inter }}$, new unknowns are required - unless all state parameters for $\boldsymbol{T}_{\text {inter }}$ could be extracted from those of $\boldsymbol{T}_{1}:=\boldsymbol{T}_{\text {chg }}$ and $\boldsymbol{T}_{2}:=\boldsymbol{T}_{\text {field }} \cdot{ }^{4}$ Hence, the system [(5), (31), (32)] made of Maxwell's first group and the dynamical equations for the charged medium and for the total energy tensor, cannot be closed any more. So at least one equation must be added.

4 For instance, the standard theory of mixtures makes an attempt to define $\boldsymbol{T}$ in terms of $\boldsymbol{T}_{1}, \boldsymbol{T}_{2}$, and the velocity fields $\mathbf{v}_{1}$ and $\mathbf{v}_{2}$ : Eq. (2.13) in Ref. [18]. This suggestion can be extended naturally to SET, by starting from Eq. (39) in Ref. [3] (valid for a dust), but this definition for $\boldsymbol{T}$ does not verify Eq. (32). An effective energy tensor defined in such a way would not reduce to the sum (25) in SR, as is required (see Sect. 5). 


\section{Constraints on the interaction tensor}

The effect of the gravitational field on the EM field can usually be neglected, because the gravitational force on a charged particle is extremely small as compared with the Lorentz force. When this approximation is used, the EM field is as in SR, i.e. in a flat Minkowski spacetime. There is massive experimental evidence for the predictions deduced from the flat-spacetime Maxwell equations. Therefore, it is asked here that the electrodynamics of SET should reduce to that of SR in the absence of gravitation, i.e., when the scalar gravitational field $\beta \equiv 1$ so that, accordingly [5], the physical spacetime metric $\gamma$ is the Minkowski metric. (See Eq. (45) below.) In SR, the dynamical equation for the charged medium valid in GR, Eq. (24), can be derived directly from the Lorentz 4-force (e.g. [1], Eq. (33.9)). While the dynamical equation for the EM field valid in GR, Eq. (28), is derived as it was derived above for GR, i.e. from the identity (27) and Maxwell's second group (4) (though in a simpler way for SR, with commas instead of semicolons in Cartesian coordinates; see e.g. Eq. (33.7) in Ref. [1]). Hence in SR as well as in GR:

$$
T_{\text {chg; } ; v}^{\mu \nu}+T_{\text {field ; }}^{\mu \nu}=0 .
$$

Again for SR and GR, this is compatible with the additivity assumption (25): the latter plus the dynamical equation (2) for the total energy tensor imply (39). Conversely, starting from the general decomposition (38) of the total tensor, then under the validity of Eq. (2), (39) is equivalent to

$$
T_{\text {inter ; }}^{\mu \nu}=0 .
$$

Note that Eq. (2) does apply in SET for a constant gravitational field: the latter means $\beta_{, 0}=0$, hence $g_{i j, 0}=0$ in (30) (see Eq. (45) below), hence $b^{\mu}=0$ in (32). Eq. (2) does apply in SET in the absence of gravitation, i.e., when $\beta \equiv 1$. Thus, if Eq. (39) of SR is indeed recovered from SET in the absence of gravitation, then Eq. (40) will apply in that situation. Below, to confirm the form of the interaction energy tensor, it is assumed that Eq. (40) applies to SET in the absence of gravitation, and in Sect. 6 it is shown that this is the case with the adopted framework.

From above, it is not a priori obvious that the condition that the interaction tensor $\boldsymbol{T}_{\text {inter }}$ should vanish in SR may be imposed. Instead, the requirement that it should be Lorentz-invariant in SR may be imposed. Any Lorentzinvariant second-order tensor is a scalar multiple of the Minkowski metric tensor, $\gamma^{0}$ [19]. Therefore, $\boldsymbol{T}_{\text {inter }}$ should have the form (in Cartesian coordinates for the Minkowski metric, i.e., $\left.\left(\gamma^{0}\right)_{\mu \nu}=\eta_{\mu v}\right)$ :

$$
T_{\text {inter } \mu \nu}=p \eta_{\mu \nu} \quad \text { (SR), }
$$

with some scalar field $p$. This defines of course a Lorentzinvariant tensor field. Note that (41) is equivalent to:

$$
T_{\text {inter } v}^{\mu}:=\eta^{\mu \rho} p \eta_{\rho v}=p \delta_{v}^{\mu}
$$

(again in Cartesian coordinates). The definition

$$
T_{\text {inter } v}^{\mu}:=p \delta_{v}^{\mu},
$$

thus set in Cartesian coordinates in a Minkowski spacetime, is actually generally-covariant: if (43) applies in some coordinates $x^{\mu}$ in a general spacetime with metric $\gamma$, it still applies after any coordinate change. Therefore, for the general case the definition (43) is used, which has been obtained by requiring that $\boldsymbol{T}_{\text {inter }}$ be Lorentz-invariant in SR. When gravitation is absent (i.e. when the metric turns out to be Minkowski's) then we expect that Eq. (40) should apply, and this implies $p=$ Constant, say $p \equiv p_{\infty}$. Indeed, if the metric is Minkowski's, Eqs. (40) and (41) imply that $p_{, \mu}=0$. Thus by requiring only that $\boldsymbol{T}_{\text {inter }}$ should be Lorentz-invariant in SR, it is then constant. Moreover, it is natural to assume that, very far from any body, the total energy tensor $\boldsymbol{T}$, as well as $\boldsymbol{T}_{\mathrm{chg}}$ and $\boldsymbol{T}_{\text {field }}$, are zero. That assumption implies that the constant $p_{\infty}$ is zero, and hence that the additivity condition (25) applies in SR. Also, with the definition (43), there is just one additional unknown $(p)$ in the system of electrodynamics of SET [(5), (31), (32)], see the end of Sect. 3. Thus one additional scalar equation is required. Therefore the standard Maxwell second group (4) cannot be used, since it would add four independent scalar equations. This is an important point, hence it is now discussed in detail.

Once an interaction tensor is introduced through Eq. (38), it may a priori be postulated that the standard Maxwell second group (4) applies, in addition to the system of electrodynamics of SET [(5), (31), (32)] [4]. This leads to (Eq. (104) in Ref. [4]):

$$
T_{\text {inter } ; \nu}^{\mu v}-b^{\mu}\left(\boldsymbol{T}_{\text {inter }}\right)=b^{\mu}\left(\boldsymbol{T}_{\text {field }}\right) .
$$

Now compute the 1.h.s. (this will be used also in the next section). Until the end of this section, and again in Sect. 7 , we will use coordinates $x^{\mu}$ that are adapted to the preferred reference fluid $\varepsilon$ and that, moreover, are such that the spatial coordinates are Cartesian for the Euclidean spatial metric $\mathbf{g}^{0}$ of the theory ( $\mathbf{g}^{0}$ is time-independent in any coordinates that are adapted to the preferred frame $\varepsilon$ ). In addition, the time coordinate will be $x^{0}=c T$ with $T$ the preferred time of the theory. In such coordinates, the curved "physical" spacetime metric $\gamma$ is [5]:

$$
\mathrm{d} s^{2}=\gamma_{\mu \nu} \mathrm{d} x^{\mu} \mathrm{d} x^{\nu}=\beta^{2}\left(\mathrm{~d} x^{0}\right)^{2}-g_{i j} \mathrm{~d} x^{i} \mathrm{~d} x^{j}
$$




$$
=\beta^{2}\left(\mathrm{~d} x^{0}\right)^{2}-\beta^{-2} \mathrm{~d} x^{i} \mathrm{~d} x^{i} .
$$

This implies that, in such coordinates

$$
\gamma:=\operatorname{det}\left(\gamma_{\mu \nu}\right)=-\beta^{-4} \text {. }
$$

Using an identity for $T_{\mu}{ }^{v} ; v$ ([1], Eq. (86.11)) and the definition (30) of $b^{\mu}$, results in (for whatever symmetric tensor $\left.T_{\mu v}\right)$ :

$$
\begin{aligned}
T_{0 ; \nu}^{v}-b_{0}(\boldsymbol{T}) & \equiv \beta^{2}\left(T_{, 0}^{00}+T_{, j}^{0 j}\right)-\beta \beta_{, 0} T^{00}, \\
T_{i ; \nu}^{\nu}-b_{i}(\boldsymbol{T}) & \equiv \beta^{2}\left(\frac{T_{i}^{v}}{\beta^{2}}\right)_{, v}-\beta^{-3} \beta_{, 0} T^{0 i} \\
& -\beta^{-3} \beta_{, i} T^{j j}-\beta \beta_{, i} T^{00} .
\end{aligned}
$$

The definition adopted (43) for $\boldsymbol{T}_{\text {inter }}$, provides:

$$
\begin{gathered}
\delta_{0}=p_{, 0}-3 p \beta,{ }_{, 0} \beta^{-1}, \\
\delta_{i}=p_{, i},
\end{gathered}
$$

where

$$
\delta_{\mu}:=\delta_{\mu}(p):=T_{\text {inter } \mu ; \nu}^{v}-b_{\mu}\left(\boldsymbol{T}_{\text {inter }}\right) .
$$

Equation (44) is equivalent to

$$
\delta_{\mu}(p)=b_{\mu}\left(\boldsymbol{T}_{\text {field }}\right) \quad(\mu=0, \ldots, 3) .
$$

For a general EM field, $b_{\mu}\left(\boldsymbol{T}_{\text {field }}\right)$ can be any 4-vector field. (This can be seen by using the explicit forms of $\boldsymbol{T}_{\text {field }}$ and $b^{\mu}$, Eqs. (26) and (30).) Hence, Eqs. (49) and (50) imply that there cannot in general exist a scalar field $p$ that verifies the four equations (52): the three spatial equations ( $\mu=$ $i=1,2,3)$ imply that $\operatorname{rot}\left(b_{i}\right)=\mathbf{0}$, which is not generally true.

Thus, postulating the validity of the standard Maxwell second group (4) would require a more general form than (43) for the interaction tensor $\boldsymbol{T}_{\text {inter, }}$, which instead should depend on four scalar fields. In that case, the interaction tensor would not be Lorentz-invariant in SR any more. It is unclear whether or not it would then be possible to get the constancy of $\boldsymbol{T}_{\text {inter }}$ in SR - which applies to the singlescalar form (43) as will be shown in the next section.

\section{Charge conservation and determination of the interaction tensor}

With the general decomposition (38), the dynamical equation (32) for the total energy tensor in SET is equivalent to:

$$
T_{\text {field ; }}^{\mu \nu}=b^{\mu}\left(\boldsymbol{T}_{\text {field }}\right)+b^{\mu}\left(\boldsymbol{T}_{\text {chg }}\right)-T_{\text {chg; } ; v}^{\mu \nu}
$$

$$
+b^{\mu}\left(\boldsymbol{T}_{\text {inter }}\right)-T_{\text {inter ; }}^{\mu \nu} \cdot
$$

By using the identity (27) for $T_{\text {field } ; v}^{\mu \nu}$, the dynamical equation (31) for the charged medium, and the definition (51), this rewrites as

$$
F_{\lambda}^{\mu} F_{; \nu}^{\lambda v}=\mu_{0}\left[b^{\mu}\left(\boldsymbol{T}_{\text {field }}\right)-F^{\mu}{ }_{v} J^{v}-\delta^{\mu}(p)\right] .
$$

If the matrix $\left(F_{\lambda}^{\mu}\right)$ is invertible, which is the generic situation and is equivalent to $\mathbf{E . B} \neq 0$ [4], this can be rewritten as

$$
F_{; v}^{\mu \nu}=\mu_{0}\left[G^{\mu}{ }_{v}\left(b^{v}\left(\boldsymbol{T}_{\text {field }}\right)-\delta^{v}(p)\right)-J^{\mu}\right],
$$

where $\left(G^{\mu}{ }_{v}\right)$ is the inverse matrix of matrix $\left(F^{\mu}{ }_{v}\right)$. By using the identity (13), one gets from this:

$$
J_{; \mu}^{\mu}=\left[G^{\mu}{ }_{v}\left(b^{v}\left(\boldsymbol{T}_{\text {field }}\right)-\delta^{v}(p)\right)\right]_{; \mu} .
$$

Apart from the identities (27) and (13) (which are valid independently of any physical theory), the validity of Eqs. (55) and (56) depends only on the validity of Eqs. (32) and (31), accounting for the general decomposition (38) with the interaction energy tensor (43). In Sect. 5, it was shown that, to close the system of electrodynamics of SET [(5), (31), (32)] in the presence of the interaction energy tensor (43), just one additional scalar equation is required. Therefore, the system can be closed by adding the conservation of charge $J_{; \mu}^{\mu}=0$, i.e., in view of (56), by adding the following equation:

$$
\left[G^{\mu \nu}\left(b_{v}\left(\boldsymbol{T}_{\text {field }}\right)-\delta_{v}(p)\right)\right]_{; \mu}=0 .
$$

Thus, we have the system [(5), (31), (32), (57)], or equivalently the system [(5), (31), (55), (57)], which has the differential identity (12). Therefore there are $13-1=12$ equations for 12 unknowns $F_{\mu \nu}(0 \leq \mu<v \leq 3), \mathrm{J}, \rho^{\star}$, and $p$. It is natural to expect that the system [(5), (31), (55), (57)] has a unique solution when suitable boundary conditions are imposed, as has the system of electrodynamics of a metric theory, [(4)-(5), (24)]. (As at the end of Sect. 3, the fact that the scalar gravitational field $\beta$ is also unknown can be accounted for by adding the scalar flat-spacetime wave equation for $\psi:=-\log \beta$.)

Now consider the case of a constant gravitational field, which includes the situation without any gravitational field. In that case, the general dynamical equation of GR (2) applies to SET as noted after Eq. (40), so that from the general decomposition (38) and the definition (43) of the interaction tensor the following is derived:

$$
\begin{aligned}
T_{; \nu}^{\mu \nu}=0 & =T_{\text {field } ; v}^{\mu \nu}+T_{\text {chg } ; v}^{\mu \nu}+T_{\text {inter } ; v}^{\mu \nu} \\
& =T_{\text {field } ; v}^{\mu \nu}+T_{\text {chg } ; v}^{\mu \nu}+p_{, v} \gamma^{\mu \nu} .
\end{aligned}
$$

Hence, the system [(5), (31), (55), (57)] is solved by $p=$ Constant : $=p_{\infty}$ and with the fields $\boldsymbol{F}, \mathbf{J}, \rho^{\star}$ being the 
solution of the system [(4)-(5), (24)] for the given timeindependent metric and for the relevant boundary conditions. Therefore, Eq. (40) is true in a constant gravitational field. In particular, Eq. (40) is true when the latter vanishes - as provisionally assumed after postulating the form (43) of the interaction energy tensor. Moreover, as noted after Eq. (43), we may assume that the constant $p_{\infty}$ is zero, hence the additivity (25) of the energy tensors $\boldsymbol{T}_{\text {chg }}$ and $\boldsymbol{T}_{\text {field }}$ does apply in a constant gravitational field.

If the EM field $\boldsymbol{F}$ and the gravitational field $\beta$ are considered given, then the scalar field $p$, and hence the interaction energy tensor, may in principle be calculated by solving the equation for charge conservation, Eq. (57). In addition $\boldsymbol{F}$ and $\beta$ are coupled with the other fields that include $p$.

\section{First approximation of the scalar field in a weak gravitational field}

In this section equations will be derived that should allow the future numerical assessment of the scalar field $p$ and the interaction energy in a given EM field and a given weak and slowly varying gravitational field. To achieve this, the results of the asymptotic framework developed in some detail in Sects. 4 and 5 of Ref. [4] will be used. The only change with respect to that former work is the additional term $-G^{\mu v} \delta_{v}(p)$ in Eq. (55), as compared with Eq. (22) in Ref. [4], and correspondingly the additional equation (57). With the additional equation (57), it is required that $\delta_{v}(p)$ be of the same order as is $b_{v}\left(\boldsymbol{T}_{\text {field }}\right)$ in the gravitational weak-field parameter $\lambda$, with $\lambda=c^{-2}$ in specific $\lambda$-dependent units of mass and time. From Eqs. (34) and (36) in Ref. [4], for the order of $b_{v}$ :

$$
b_{v}\left(\boldsymbol{T}_{\text {field }}\right)=\operatorname{ord}\left(c^{2 n-5}\right),
$$

where

$$
\boldsymbol{F}=c^{n}\left(\stackrel{0}{\boldsymbol{F}}+c^{-2} \stackrel{1}{\boldsymbol{F}}+O\left(c^{-4}\right)\right)
$$

is the expansion of the EM field tensor. The gravitational field is expanded as (Eq. (28) in Ref. [4]):

$$
\beta:=\sqrt{\gamma_{00}}=1-U c^{-2}+O\left(c^{-4}\right),
$$

where $U$ is the Newtonian gravitational potential. Using this and setting

$$
p=c^{q}\left(\stackrel{0}{p}+c^{-2} \stackrel{1}{p}+O\left(c^{-4}\right)\right)
$$

from (49) and (50) it is shown that $\delta_{\mu}=\operatorname{ord}\left(c^{q}\right)$, hence the requirement is satisfied iff $q=2 n-5$ so that

$$
p_{, \mu}=c^{2 n-5}\left(\stackrel{0}{p}_{, \mu}+O\left(c^{-2}\right)\right) .
$$

Let us define

$$
\hat{\rho}:=\left(G^{\mu}{ }_{\nu} b^{v}\left(\boldsymbol{T}_{\text {field }}\right)\right)_{; \mu},
$$

as in Eq. (23) in Ref. [4] - but now $\hat{\rho} \neq J_{; \mu}^{\mu}$, unlike in the latter work. With this definition, everything in Sects. 4 and 5 of Ref. [4] remains valid and we have in particular (Eq. (45) in Ref. [4]): ${ }^{5}$

$$
\begin{aligned}
\hat{\rho} & =c^{-3}\left[\left(G_{1}{ }^{\mu 0} T_{1}{ }^{j j}-G_{1}{ }^{\mu i} T_{1}{ }^{0 i}\right) \partial_{T} U\right]_{, \mu}(1 \\
& \left.+O\left(c^{-2}\right)\right),
\end{aligned}
$$

where $G_{1}{ }^{\mu \nu}$ and $T_{1}{ }^{\mu \nu}$ are the first approximations of $G^{\mu \nu}$ and $T^{\mu \nu}$, i.e.,

$$
\boldsymbol{G}_{1}:=\left(\boldsymbol{F}_{1}\right)^{-1}, \quad \boldsymbol{F}_{1}:=c^{n} \boldsymbol{F},
$$

and the like for $\boldsymbol{T}_{1}$ [4]. This can be calculated: as given by Eqs. (53) and (54) of Ref. [4],

$$
\hat{\rho}=c^{-3}\left(e^{i} \partial_{T} U\right)_{, i}\left(1+O\left(c^{-2}\right)\right),
$$

where

$$
\begin{gathered}
e^{i}=\frac{1}{2 c \mu_{0}\left(B_{1} E_{1}+B_{2} E_{2}+B_{3} E_{3}\right)} \times \\
\left(\begin{array}{c}
c^{2}\left(B_{1}{ }^{3}+B_{1} B_{2}{ }^{2}+B_{1} B_{3}{ }^{2}\right)+B_{1} E_{1}{ }^{2}-B_{1} E_{2}{ }^{2}-B_{1} E_{3}{ }^{2} \\
+2 B_{2} E_{1} E_{2}+2 B_{3} E_{1} E_{3} \\
c^{2}\left(B_{2}{ }^{3}+B_{2} B_{3}{ }^{2}+B_{2} B_{1}{ }^{2}\right)+B_{2} E_{2}{ }^{2}-B_{2} E_{3}{ }^{2}-B_{2} E_{1}{ }^{2} \\
+2 B_{3} E_{2} E_{3}+2 B_{1} E_{2} E_{1} \\
c^{2}\left(B_{3}{ }^{3}+B_{3} B_{1}{ }^{2}+B_{3} B_{2}{ }^{2}\right)+B_{3} E_{3}{ }^{2}-B_{3} E_{1}{ }^{2}-B_{3} E_{2}{ }^{2} \\
+2 B_{1} E_{3} E_{1}+2 B_{2} E_{3} E_{2}
\end{array}\right)
\end{gathered}
$$

In Eq. (68), $E_{i}:=E^{i}$ and $B_{i}:=B^{i}$ are the components of the first approximations of the electric and magnetic fields in the frame $\mathcal{E}$, in coordinates of the class specified after Eq. (44). I.e., $E^{i}$ and $B^{i}$ are extracted (Note 2) from the first approximation $\boldsymbol{F}_{1}$ of the EM field tensor $\boldsymbol{F}$, Eq. (66), that obeys the flat-spacetime Maxwell equations [4]. Inserting (63) and (67) into (57) using $u_{; \mu}^{\mu}=\left(u^{\mu} \sqrt{-\gamma}\right), \mu / \sqrt{-\gamma}$, with $\sqrt{-\gamma}=1+O\left(c^{-2}\right)$ owing to (46) and (61), provides:

$$
\left(G_{1}^{\mu \nu}\left(p_{1}\right)_{, v}\right)_{, \mu}=G_{1}^{\mu \nu}{ }_{, \mu}\left(p_{1}\right)_{, v}
$$

5 The remainder in this expansion was incorrectly written as $O\left(c^{-5}\right)$ in Eqs. (45) and (54) of Ref. [4]. In fact it is $O\left(c^{n-7}\right)$ while the main term is ord $\left(c^{n-5}\right)$, Eq. (41) of Ref. [4]. The important point is precisely that the remainder is $O\left(c^{-2}\right)$ times the main term. 


$$
=c^{-3}\left(e^{i} \partial_{T} U\right)_{, i}\left(1+O\left(c^{-2}\right)\right)
$$

where $p_{1}:=c^{2 n-5} \stackrel{0}{p}$ is the first approximation of $p$. The first equality is due to the antisymmetry of $G^{\mu \nu}$ and $G_{1}{ }^{\mu \nu}$. The matrix $\boldsymbol{G}^{\prime}:=\left(G_{1}{ }^{\mu \nu}\right)$ is given explicitly by Eq. (50) in Ref. [4], which can be rewritten as

$$
\boldsymbol{G}^{\prime}=\frac{-c}{\mathbf{E} . \mathbf{B}} \boldsymbol{H}, \quad \boldsymbol{H}:=\left(\begin{array}{cccc}
0 & B_{1} & B_{2} & B_{3} \\
-B_{1} & 0 & -\frac{E_{3}}{c} & \frac{E_{2}}{c} \\
-B_{2} & \frac{E_{3}}{c} & 0 & -\frac{E_{1}}{c} \\
-B_{3} & -\frac{E_{2}}{c} & \frac{E_{1}}{c} & 0
\end{array}\right) .
$$

It is easy to check that Maxwell's (flat-spacetime) first group, verified by $\boldsymbol{F}_{1}$, implies that

$$
H_{, \mu}^{\mu \nu}=0 .
$$

It follows from this and (70) that in (69):

$$
\begin{aligned}
& \left(k^{v}\right):=\left(G_{1}{ }^{\mu \nu}, \mu\right)= \\
& \frac{1}{(\mathbf{E . B})^{2}} \times\left(\begin{array}{c}
-B_{i} c(\mathbf{E . B})_{, i} \\
E_{3}(\mathbf{E . B})_{, 2}-E_{2}(\mathbf{E . B})_{, 3}+B_{1} c(\text { E.B })_{, 0} \\
E_{1}{\text { (E.B) }{ }_{, 3}-E_{3}(\text { E.B })_{, 1}+B_{2} c(\text { E.B })_{, 0}}_{E_{2}(\text { E.B })_{, 1}-E_{1}(\text { E.B })_{, 2}+B_{3} c(\text { E.B })_{, 0}}
\end{array}\right),
\end{aligned}
$$

i.e.,

$$
\begin{gathered}
k^{0}=\frac{-c}{(\mathbf{E} . \mathbf{B})^{2}} \mathbf{B} \cdot(\nabla(\mathbf{E} . \mathbf{B})), \\
\left(k^{i}\right)=\frac{1}{(\mathbf{E} . \mathbf{B})^{2}}\left(\frac{\partial(\mathbf{E} . \mathbf{B})}{\partial T} \mathbf{B}-\mathbf{E} \wedge(\nabla(\mathbf{E} . \mathbf{B}))\right) .
\end{gathered}
$$

Equation (69) can be rewritten in the form

$$
\partial_{T} p_{1}+u^{j} \partial_{j} p_{1}=S
$$

where

$$
S:=\frac{c^{-2}\left(e^{i} \partial_{T} U\right)_{, i}}{k^{0}}
$$

(no confusion can occur with the sum $S$ in Subsect. 2.1), and

$$
u^{j}:=\frac{c k^{j}}{k^{0}}
$$

We assume that $k^{0} \neq 0$ in Eq. (72), i.e. B. $(\nabla($ E.B $)) \neq 0$. Note that $\mathbf{E . B} \neq 0$ is required from Sect. 6. Note that here the first-approximation fields $\mathbf{E}$ and $\mathbf{B}$ are involved, and they obey the flat-spacetime Maxwell equations. Equation (75) is an advection equation with a given source $S$ for the unknown field $p_{1}$. This is a hyperbolic PDE whose characteristic curves are the integral curves of the vector field $\mathbf{u}:=\left(u^{j}\right)$. That is, on the curve $\mathcal{C}\left(T_{0}, \mathbf{x}_{0}\right)$ defined by

$$
\frac{\mathrm{d} \mathbf{x}}{\mathrm{d} T}=\mathbf{u}(T, \mathbf{x}), \quad \mathbf{x}\left(T_{0}\right)=\mathbf{x}_{0},
$$

we have from (75):

$$
\frac{\mathrm{d} p_{1}}{\mathrm{~d} T}=\frac{\partial p_{1}}{\partial T}+\frac{\partial p_{1}}{\partial x^{j}} \frac{\mathrm{d} x^{j}}{\mathrm{~d} T}=S(T, \mathbf{x})
$$

Note that the field $\mathbf{u}$ is given, Eq. (77), since it does not depend on the unknown field $p_{1}$. Therefore, the integral lines (78) are given, too, hence the characteristic curves do not cross. Thus, the solution $p_{1}$ is obtained unambiguously by integrating (79):

$$
p_{1}(T, \mathbf{x}(T))-p_{1}\left(T_{0}, \mathbf{x}_{0}\right)=\int_{T_{0}}^{T} S(t, \mathbf{x}(t)) \mathrm{d} t,
$$

where $T \mapsto \mathbf{x}(T)$ is the solution of (78). If at time $T_{0}$ the position $\mathbf{x}_{0}$ in the frame $\varepsilon$ is far enough from material bodies, it can be assumed that $p_{1}\left(T_{0}, \mathbf{x}_{0}\right)=0$.

\section{Conclusion}

The main results obtained in this paper are as follows:

1. The structure of classical electrodynamics based on the standard Maxwell equations of SR or GR has been discussed and it has been shown by using the notion of differential identity that the number of independent scalar PDE's is the same as the number of unknown fields. This applies to both the case with given 4-current and the more general case taking into account the equation of motion of the charged medium, the 4-current then belonging to the unknowns. The result for the first case is well known (e.g. $[7,8])$, but the explanation by considering the differential identities (12) and (15) is more straightforward and was not identified in preceding literature. For the more general case also, the discussion is based on differential identities: (12) is still valid but (15) holds now only on the solution space; this discussion also appears to be new.

2. In the investigated theory of gravity ("SET"), with the additivity assumption (25), electrodynamics consists of the system [(5), (31), (32)], i.e., Maxwell's first group and the dynamical equations for the charged medium and for the total energy tensor. Using the same method as for SR and GR, it has been shown that this is also a closed system of PDE's. While introducing the "interaction energy tensor" $\boldsymbol{T}_{\text {inter }}$ by switching to (38), it is necessary to introduce new unknowns, so that the foregoing system is no longer closed and new equations are required. For SR $\boldsymbol{T}_{\text {inter }}$ is required to be a Lorentz-invariant tensor. This determines that in the general case it has 
the form $T_{\text {inter } v}^{\mu}:=p \delta_{v}^{\mu}$, with $p$ a scalar field (which is constant and zero in SR). Thus, only one additional equation is needed and this can consistently be imposed to be the charge conservation.

3. Considering a weak and slowly varying gravitational field, we derived the equation that determines the scalar field $p$, whose knowledge is equivalent to that of the interaction energy tensor: Eq. (75). It was shown how this may in principle be used to compute that field in a given EM field and in a given weak and slowly varying gravitational field, Eq. (80). The interaction energy is gravitationally active, because its density $T_{\text {inter }}^{00}=p \gamma^{00}$ contributes to the total energy density of matter and non-gravitational fields, $T^{00}$. According to SET [5], $T^{00}$ is the source of the gravitational field. The interaction energy is not localized inside matter, and it has to be present in space as soon as there is matter that is electromagnetically active. It could thus be counted as "dark matter". To learn more, it will be necessary to have recourse to a numerical work.

Acknowledgement: I am grateful to Jerzy Kijowski for a discussion on the motion of the sources. Also, a referee asked for more explanation about why the interaction energy could contribute to dark matter.

\section{References}

[1] Landau L.D., Lifshitz E.M., The classical theory of fields, 3rd English edition, Pergamon, Oxford, U.K., 1971

[2] Jackson J.D., Classical electrodynamics (3rd edn), Wiley, Hoboken (N.J.), U.S.A., 1998

[3] Arminjon M., Continuum dynamics and the electromagnetic field in the scalar ether theory of gravitation, Open Physics, 2016, 14, 395-409.
[4] Arminjon M., Charge conservation in a gravitational field in the scalar ether theory, Open Physics, 2017, 15, 877-890.

[5] Arminjon M., Space isotropy and weak equivalence principle in a scalar theory of gravity, Braz. J. Phys., 2006, 36, 177-189.

[6] Misner C.W., Thorne K.S., Wheeler J.A., Gravitation, Freeman, San Francisco, U.S.A., 1973

[7] Jiang B.N., Wu J., Povinelli L.A., The origin of spurious solutions in computational electromagnetics, J. Comput. Phys., 1996, 125, 104-123.

[8] Zhou X.L., On independence, completeness of Maxwell's equations and uniqueness theorems in electromagnetics, Prog. Electromagn. Res., 2006, 64, 117-134.

[9] Liu C., Explanation on overdetermination of Maxwell's equations, Physics and Engineering (in Chinese), 2017, 27(3), 7-9, and Preprint arXiv:1002.0892v9 (2018).

[10] Das A., The special theory of relativity - a mathematical exposition, 2nd printing, Springer-Verlag, Berlin - Heidelberg - New York, 1996

[11] Cattaneo C., General relativity: relative standard mass, momentum, energy and gravitational field in a general system of reference, Nuovo Cim., 1958, 10, 318-337.

[12] Arminjon M., On continuum dynamics and the electromagnetic field in the scalar ether theory of gravitation, J. Phys. Conf. Ser., 2017, 845, 012014 (9 pages).

[13] Fock V.A., The theory of space, time and gravitation, 2nd English edition, Pergamon, Oxford, U.K., 1964

[14] Chandrasekhar S., Conservation laws in general relativity and in the post-Newtonian approximations, Astrophys. J., 1969, 158, 45-54.

[15] Arminjon M., On the extension of Newton's second law to theories of gravitation in curved space-time, Arch. Mech., 1996, 48, 551-576.

[16] Arminjon M., On the possibility of matter creation/destruction in a variable gravitational field, Analele Universit. Bucureşti Fizică, 1998, 47, 3-21.

[17] Møller C., The theory of relativity, Clarendon Press, Oxford, U.K., 1952

[18] Müller I., A thermodynamic theory of mixtures of fluids, Arch. Rational Mech. Anal., 1968, 28, 1-39.

[19] Arminjon M., Lorentz-invariant second-order tensors and an irreducible set of matrices, submitted for publication, and preprint HAL-01797592, 2018. 